\title{
Assessment of Dose Perturbations for Metal Stent in Photon and Proton Radiotherapy Plans for Hepatocellular Carcinoma
}

\section{Boram Lee}

Seoul National University Bundang Hospital

\section{Sungkoo Cho}

Samsung Medical Center

Hee Chul Park

Samsung Medical Center

\section{Sang-Won Kang}

Seoul National University Bundang Hospital

Jae-Sung Kim

Seoul National University Bundang Hospital

Jin-Beom Chung ( $\nabla$ jbchung1213@gmail.com )

Seoul National University Bundang Hospital https://orcid.org/0000-0003-2668-3906

\section{Research Article}

Keywords: Metal stent, Proton, Photon, Monte Carlo simulation, Hepatocellular carcinoma

Posted Date: January 31st, 2022

DOI: https://doi.org/10.21203/rs.3.rs-1244960/v1

License: (1) This work is licensed under a Creative Commons Attribution 4.0 International License. Read Full License 


\section{Abstract}

Background: The present study aimed to investigate the dosimetric impact of metal stent for photon and proton treatment plans in hepatocellular carcinoma.

Methods: Dose perturbation caused by a metal stent included in the photon and proton treatment of hepatocellular carcinoma was evaluated with comparing Eclipse and RayStation treatment planning system (TPS) to Monte Carlo (MC). Photon and proton plans were created using anteriorposterior/posterior-anterior (AP/PA) fields using a $6 \mathrm{MV}$ beam and AP/PA fields of a wobbling beam using $150 \mathrm{MeV}$ and a $10 \mathrm{~cm}$ ridge filter. The difference in dose distributions and dosimetric parameters were compared depending on the stent's positions (the bile duct (GB) and the intestinal tract (GI)) and angles $\left(0^{\circ}, 45^{\circ}\right.$, and $\left.90^{\circ}\right)$. In addition, the dose variation in the target volume including the stent was comparatively evaluated through dose volume histogram (DVH) analysis.

Results: The percentage differences in the dosimetric parameters calculated by $\mathrm{MC}$ ranged from -7.0 to $3.9 \%$ for the photon beam and $-33.7 \%$ to $4.3 \%$ for the proton beam, depending on the angle at which the GB and GI stents were placed. A maximum difference was observed at the minimum dose (Dmin), which was shown in both photon and proton plans in the GB and GI stents deployed at an incidence angle of $90^{\circ}$. The differences in these parameters were greater in the proton plan than in photon plan. The target volume showed various dose variations depending on positions and angles of stent for both beams. Compared with the no-stent, the doses within the target volume containing the GI and GB stents for the photon beam were overestimated in the high-dose area at $0^{\circ}$, nearly equal within $1 \%$ at $45^{\circ}$, and underestimated at $90^{\circ}$. These doses to the proton beam were underestimated at all angles, and the amount of underdose to the target volume increased with the increase of the stent's angle. However, the difference was much greater with the proton plan than with the photon plan.

Conclusions: Dose perturbations within the target volume due to the presence of the metal stent were not observed in the TPS calculations for photon and proton beams, but MC were used to confirm that there are the dose variation within the target volume. The $\mathrm{MC}$ results found that delivery of the treatment beam avoiding the stent was the best method to prevent underdose of the target volume.

\section{Background}

Hepatocellular carcinoma (HCC), a malignant tumour of the liver, is one of the most difficult cancers to detect and has a poor prognosis. Depending on the patient's condition, it is treated with surgical resection, radiofrequency ablation, transarterial chemoembolization (TACE), and radiotherapy (RT) [1-3]. Among these treatments, RT has not been widely used for HCC in the past due to the high resistance of the liver to radiation, the dose error occurring in moving organs, and the side effects caused by radiation [4]. However, due to the improvement of RT technology and clinical experience, the adaptation of RT to the treatment of HCC has been increasing, and the use of volume-modulation arc therapy (VMAT), tomotherapy, and proton beam therapy (PBT) has also been increasing recently $[1,5,6]$. 
HCC patients are treated with VMAT or PBT, depending on the particular case [5]. Some of the patients treated with either of these methods are treated with a stent inserted into the body during RT. The stent is generally used for bile duct dilatation, portal vein, and duodenal stenosis among the treatment sites, and the type and silicone coating is determined depending on the treatment site and the condition of patient. Stents made from a variety of materials, including nickel, titanium, iron, hafnium, copper, boron, and niobium, and using high atomic number (High-Z) materials can affect the amount of radiation delivered to the surrounding tissue [7]. Self-expanding metal stents (SEMS), which are usually applied to the treatment of disease-induced stenosis, have the advantage of acting as fiducial markers that increase the accuracy of high-dose treatments [8]. Because this type of stent is not a high-Z material such as gold, it does not generate strong artefacts and is useful as a fiducial marker for image-guided therapy [9]. Fiducial markers that enable accurate positioning have an advantage in reducing the target margin [10]. However, if the stent is placed in the pathway of the radiation field, it causes dose perturbations $[8,11-$ 13]. The dose perturbation for the photon beam is primarily due to secondary electrons and scattering caused by the metallic components within the stent [7]. Metal stents developed using nitinol, an alloy of nickel and titanium, have been clinically tested and are the most widely used $[7,8,10,13,14]$. Even in proton therapy, the dose perturbations caused by metal stents will result in underdose and overdose in that part of the target volume containing the metal stent due to secondary electrons and multi-scattering. Therefore, the range uncertainty will require additional margins when creating a treatment plan. Currently, a method used mainly in the treatment of patients with SEMS in clinical practice is to override the stent to the surrounding tissue. It is very difficult to accurately calculate the effect of the stent on the dose distribution with a treatment planning system (TPS). The reasons are as follows. First, it is difficult to define a physical area affected by the stent. The minimum voxel size reconstruction by computed tomography (CT) is approximately $1 \mathrm{~mm} 3$; however, the thickness of the stent used in the clinic is 0.16 $\mathrm{mm}$, which is relatively small, resulting in image artefacts such as blurring [10]. In addition, the TPSs that are used possess a limited ability to define regions of interest (ROIs), making it impossible to define a small stent volume. Second, due to the limitations of the TPSs, the reconstructed physical density of the stent voxel is averaged with the surrounding values and thus is evaluated to be lower than the density of the actual stent. To eliminate these problems, clinics mainly perform dose calculations by replacing the density of the stent with the density of the surrounding tissue. However, it is necessary to accurately recognise the effect of the dose perturbation caused by the stent. Several previous studies have been conducted to verify the effect of metal stents on the dose $[7,11,12,15]$. The effects of the stents were mainly studied for RT using a photon beam. In RT using a proton beam, the effects were evaluated through Monte Carlo (MC) simulations and through measurements using films. These studies were performed to verify the range of the differences in a specific area. Only a few studies focused on the dosimetric evaluation of the effects that occur in the volume where the stent is inserted.

This study aimed to understand the volumetric effect of dose perturbation that is caused by metal stents applied in HCC RT. An MC simulation was performed for both photon beams and proton beams used in actual clinical practice [16]. The metal stents (such as bile duct and gastrointestinal tract stents) applied in HCC patients were used in this study. The incident directions of the beams made angles of $0^{\circ}, 45^{\circ}$, and 
$90^{\circ}$, respectively, and the dose coverage delivered to the target volume was evaluated as a function of the angle at which the stent was positioned.

\section{Methods}

Film dosimetry is often used to evaluate the effects of radiation distribution by artefacts during RT [7]. However, it is difficult to evaluate the distribution three-dimensionally in a proton beam because of the energy dependence of the film. In addition, various measuring methods such as a thermoluminescent dosimeter (TLD), optically stimulated luminescent dosimeter (OSLD), and chamber are also widely used as a method of measuring the change in dose perturbations, but three-dimensional measurement has limitations. In this study, dose perturbation caused by metal stents was evaluated using MC simulations and a TPS for RT using photon beam and proton beam. First, CT data were obtained with a $1.25 \mathrm{~mm}$ thick slice of a water-equivalent solid phantom containing a metal stent for the evaluation of the TPS dose calculations. Second, MC simulations were performed to evaluate the dose perturbation due to the metal stent in three dimensions [8]. Geant4 (v10.3) and GATE (v8.1), which have the advantages of simple geometric configuration and fast computation time, were used for the $\mathrm{MC}$ configuration. Nitinol, an alloy of nickel and titanium currently used in clinical practice, was used for the metal stent.

Component data for the metal stents were provided by the manufacturer. The metal stent used in clinical practice and the modelled stent used in the MC simulation for dose calculation are shown in Figure 1. The stent used for evaluating the dose was constructed as follows. The mass density of the nitinol was $6.8 \mathrm{~g} / \mathrm{cm} 3$. Two types of stents were used to determine the effect of the stent size: one in the bile duct (the GB stent) and another in the gastrointestinal tract (the GI stent). The diameter of the metal wire constituting the metal stent was $0.16 \mathrm{~mm}$; when constructed in cross-section, the stent consisted of 28 wires for the bile duct and 36 wires for the gastrointestinal tract. The material composition was the same for the bile duct and the gastrointestinal tract, and the diameter of each stent was between $1 \mathrm{~cm}$ and 2 $\mathrm{cm}$ when not inserted into the body.

A Truebeam (Varian Medical Systems) linear accelerator and a proton therapy system (Sumitomo Heavy Industries Ltd.) were used to study the dose difference caused by metal stents in a photon beam and in a proton beam, respectively. The virtual machine system completed in previous studies [16-18] was used as the equipment modelling step of the MC calculation. We checked the percent depth dose and dose profile consistency for various energies to validate the Monte Carlo of the equipment in the previous studies, and used the phase space file were formatted according to recommendation of the International Atomic Energy Agency (IAEA) that confirmed the agreement with a mean error of maximum $0.5 \%$. Also, a compact cluster was designed for Monte Carlo calculation [19]. A planning schematic diagram of the configuration of the anterior-posterior/posterior-anterior (AP/PA) fields to evaluate the effect of dosimetric paratmers such as the maximum dose $\left(D_{\text {max }}\right)$, the minimum dose $\left(D_{\text {min }}\right)$, and the mean dose $\left(D_{\text {mean }}\right)$ for target volume containing metal stent is shown in Figure 2. The photon plan was generated with the AP/PA fields technique using $6 \mathrm{MV}$ of energy, and the proton plan was created with AP/PA fields of a wobbling beam using $150 \mathrm{MeV}$ of energy and a $10 \mathrm{~cm}$ ridge filter [20]. The plans for the photon 
beam and the proton beam were both created with the same parameters in the TPS and the MC simulation. A water phantom of $20 \mathrm{~cm} \times 20 \mathrm{~cm}$ was constructed, and a metal stent was placed in the centre of the phantom. The effect of various beam angles on the stent inserted into the patient was considered. The stent was fixed at angles of $0^{\circ}, 45^{\circ}$, and $90^{\circ}$, respectively, to evaluate the angular dependence by calculating the difference in the influence depending on the incident angle of the beam [8]. The value of the range cut of the water phantom was 0.01 with respect to the size of the wire, and the dose value calculated by the DoseActor function was reconstructed into a voxel size of $1 \times 1 \times 1 \mathrm{~mm}^{3}$ voxel size. The physics list library for the calculation of the photon and proton beams used the QuarkGluonStrongG4Precompound-BInaryCascade-HighPrecision neutron-ElectroMagnetic opt Z (QGSPBIC-HP-EMZ) reference physics list [21].

The calculated results were sent to a system that had been programmed to be converted into a digital imaging and communications in medicine (DICOM) format compatible with the TPS. Since the digital value in the uncorrected simulation required a tool for analysis, we set the configuration to import the frame ID value into the TPS and performed the dose analysis using the TPS function. The output values calculated in the MC simulation were compared and analyzed as relative values. For evaluation, a contour for dose evaluation was made by expanding by $5 \mathrm{~mm}$, based on the metal stent. Dose volume histograms (DVHs) were used to compare the differences according to the type of radiation and the shape and size of the metal stent.

\section{Results}

The dose distributions calculated from TPSs for the photon and proton beams suing phantom images with and without metal stents are shown in Figure 3. As shown in Figure 3, there were no dose perturbations in the area containing the metal stent in the photon and proton plans obtained from the TPS. This means that TPS calculations for photon and proton beams did not accurately calculate the dose perturbation in the area containing the metal stent. The differences between the axial dose distributions calculated by the $\mathrm{MC}$ simulations for the photon and proton beams, with and without the stent, are shown in Figure 4 and 5. As shown in these figures, it was visually confirmed that the dose was changed compared to the dose distribution calculated without the stent due to the change in the stent size depending on the various position angles $\left(0^{\circ}, 45^{\circ}\right.$, and $\left.90^{\circ}\right)$ of the stent.

The differences in the values of the dosimetric parameters calculated in the $\mathrm{MC}$ simulation corresponding to the various stent positions and angles, compared to the values without a stent, are listed in Table 1 and 2. For the photon beam, the values of $D_{\max }$ when the stent was placed into the body were higher, by $0 \sim 3.9 \%$, than the value without the stent. The largest difference (3.9\%) in $D_{\max }$ was observed in the $G B$ stent positioned at $0^{\circ}$ compared with the absence of the stent. The values of $D_{\min }$ were within $1.1 \%$ of the no-stent value for the GB stent and the GI stent when the stents were placed at $0^{\circ}$ and $45^{\circ}$. The $D_{\min }$ value obtained at an angle of $90^{\circ}$ showed a relatively large difference, with decreases of $5.9 \%$ for the GB stent and $7.0 \%$ for the GI stent, respectively. The values of $D_{\text {mean }}$ were not significantly different for the stents 
positioned at $0^{\circ}$ and at $45^{\circ}$. For the stents positioned at $90^{\circ}$, there were small differences for both the GB stent $(-1.7 \%)$ and the $\mathrm{GI}$ stent $(0.7 \%)$. The differences in the dosimetric parameters (compared to not using the stent) were large for both the GB stent and the GI stent located at $90^{\circ}$.

Table 1

Percentage difference of dosimetric parameters for photon beam according to stent position and angle compared to that without a stent though MC simulation.

\begin{tabular}{|lllllll|}
\hline & $\mathbf{0}^{\circ}$ & & $\mathbf{4 5}^{\circ}$ & & $\mathbf{9 0}^{\circ}$ & \\
& GB stent & Gl stent & GB stent & Gl stent & GB stent & Gl stent \\
\hline$D_{\text {max }}(\%)$ & 3.9 & 2.4 & 0.4 & 0.0 & 1.9 & 1.7 \\
\hline$D_{\text {min }}(\%)$ & -1.1 & -1.0 & 0.2 & -0.1 & -5.9 & -7.0 \\
\hline$D_{\text {mean }}(\%)$ & 0.0 & -0.1 & -0.1 & -0.1 & -1.7 & -0.7 \\
\hline
\end{tabular}

GB stent: stent used in the bile duct, GI stent: stent used in gastrointestinal tract, $D_{\text {max }}$ : maximum dose, $D_{\text {min }}$ : minimum dose, $D_{\text {mean }}$ : mean dose

Table 2

Percentage difference of dosimetric parameters for proton beam according to stent position and angle compared to that without a stent though MC simulation.

\begin{tabular}{|lllllll|}
\hline & $\mathbf{0}^{\circ}$ & & $\mathbf{4 5}^{\circ}$ & \multicolumn{3}{l|}{$\mathbf{9 0}^{\circ}$} \\
\hline & GB stent & Gl stent & GB stent & Gl stent & GB stent & Gl stent \\
\hline$D_{\text {max }}(\%)$ & 0.7 & 0.6 & 1.4 & 1.6 & 4.3 & 2.7 \\
\hline$D_{\text {min }}(\%)$ & -6.0 & -2.7 & -11.7 & -14.7 & -33.7 & -21.9 \\
\hline$D_{\text {mean }}(\%)$ & -0.5 & -0.1 & -0.6 & -0.6 & -4.1 & -1.2 \\
\hline
\end{tabular}

GB stent: stent used in the bile duct, GI stent: stent used in gastrointestinal tract, $\mathrm{D}_{\text {max }}$ : maximum dose, $D_{\text {min }}$ : minimum dose, $D_{\text {mean }}$ : mean dose

For the proton beam, the values of $D_{\max }$ were $0.7 \sim 4.3 \%$ higher than the value without the stent because of the interaction between the proton beam and the metal stent. Compared to not using the stent, $D_{\max }$ showed the largest difference (4.3\%) for the GB stent located at $90^{\circ}$. In terms of $D_{\min }$, all values showed lower doses; the differences were greater than $6 \%$ compared with the no-stent case, except for a difference of $2.7 \%$ for the $\mathrm{GI}$ stent positioned at $0^{\circ}$. The differences in the values of $D_{\min }$ were relatively large, $-33.7 \%$ and $-21.9 \%$ in the GB and GI stents, respectively, for stent locations at $90^{\circ}$. The values of $D_{\text {mean }}$ were within $1 \%$ of the no-stent values for the $\mathrm{Gl}$ and GB stents located at $0^{\circ}$ and $45^{\circ}$ (as in the photon beam), whereas there was a slightly higher difference with the GB and GI stents located at $90^{\circ}$. 
Comparisons of the DVHs at the various position angles $\left(0^{\circ}, 45^{\circ}\right.$, and $\left.90^{\circ}\right)$ of the stent with those without the stent are shown in Figure 6 and 7. The analysis based on the DVHs enables straightforward discrimination of the dose difference with respect to the volume. In both the photon beam and the proton beam, it was observed that the dose varied with the angle of the stent as shown in Figure 6 and 7.

For the photon beam, the DVHs for the GB and GI stents positioned at $0^{\circ}$ showed a difference with respect to the volume of the high-dose region. For the GB and GI stents positioned at $45^{\circ}$, the DVHs were similar to those without the stent. In contrast, the DVHs for the $\mathrm{GI}$ and GB stents positioned at $90^{\circ}$ showed significant differences, indicating underdoses for the target volume.

For the proton beam, the DVHs indicated underdoses in the target volume for the GI and GB stents at all angles, as compared with the DVHs obtained without stents. The underestimated volume seen in the DVHs was exacerbated as the stent angle was increased. The GI and GB stents positioned at $90^{\circ}$ were accompanied by differences in the high-dose region, indicating that high-dosing increased in accordance with the use of the stent. This trend was similar for the photon beam and the proton beam; however, the difference was much larger for the proton beam than for the photon beam.

\section{Discussion}

Because the stent causes artefacts and blurring in a CT image, the areas where these blemishes occur are generally assigned with the Hounsfield unit $(\mathrm{HU})$ value of the surrounding tissue to generate a treatment plan. Previous studies using film and TLD measurements have reported that these blemishes indicate dose differences. In addition, previous studies have reported that dose changes of up to $20 \%$ could occur due to substances with different densities having been inserted into the body [22, 23]. In this study, dose fluctuations caused by metal stents used in clinical practice for photon beams and proton beams were evaluated through MC simulations. The MC simulations took into account all of the major physical processes leading to dose deposition, including mean energy loss, range straggling, multiple Coulomb scattering, and nuclear reactions.

In order to perform an appropriate experiment in this study, a metal stent was inserted into the intensity modulated radiation therapy (IMRT) phantom, and a CT scan was performed with this phantom. The wire thickness of the used stent was $0.1 \mathrm{~mm}$, which was smaller than the detector size of the CT. As a result, the stent did not appear clearly on the CT image, producing a blurry image instead. The stent density was assigned an average value corresponding to the densities of the surrounding materials, which amounted to $1.08 \sim 1.3 \mathrm{~g} / \mathrm{cm}^{3}$, a range of densities much lower than the actual density of the stent [10]. Therefore, the dose variations calculated from the TPS with the inclusion of metal stents showed little difference between the photon beam and the proton beam. The authors considered these calculated values to be inaccurate representations of reality, and we conducted an $M C$ simulation to verify the differences in dose variation. 
In order to analyse the dosimetric parameters such as $D_{\text {max }}, D_{\text {mean }}$, and $D_{\min }$, the dose enhancement was observed in $D_{\max }$ because of the multiple Coulomb scattering caused by the interactions with the highdensity material. Compared to the no-stent case, the largest difference in $D_{\max }$ was found when the stent was positioned at $0^{\circ}$ for the photon beam and at $90^{\circ}$ for the proton beam. Values of $D_{\min }$ were lower for both the GB and the GI stents positioned at all angles, except for the stents positioned at $45^{\circ}$. The value of $D_{\min }$ indicated that the degree of underdose increased as the angle of the positioned stent increased. In addition, the differences observed for the proton beam were more pronounced than those observed for the proton beam. Both the photon and proton beams showed a more significant reduction when the GB and $\mathrm{Gl}$ stents were positioned at $90^{\circ}$ than when they were positioned at $0^{\circ}$ and $45^{\circ}$. This was because both the photon and proton beams interacted more intensively with the stent when they were incident on the stents positioned at $90^{\circ}$, due to the overlapping effect. However, it is expected that the differences observed in this study will not be replicated in the clinical situation because the positioning of a stent at $90^{\circ}$ is rarely applied. The values of $D_{\text {mean }}$ for both the photon and proton beams also indicated reduced doses for the stents located at all angles, compared with those obtained without a stent. However, the dose reductions for $D_{\text {mean }}$ were not greater than those for $D_{\max }$ and $D_{\min }$.

The analysis of the DVHs indicated that there was an underdose to the target volume when the stent was positioned at the specified angles, as compared with the no-stent dose, for both the photon and proton beams. The largest dose reduction in the DVHs occurred with the stent located at $90^{\circ}$, for both the photon and proton beams. In addition, the proton beam displayed a greater dose reduction for the target volume than the photon beam. That outcome may have occurred because of the influence of the dose shadow on the proton beam [8]. A comparison of Figure 4 and 5 reveals that the proton beam had an underdose in the black colour around the stent, as compared with the photon beam. The calculated results of the MC simulation were evaluated with a statistical uncertainty that lay within $1 \%$ for all voxels.

This study had two limitations. One limitation was that the stent modelling for the MC calculation consisted of only nitinol wire. However, the tip of the nitinol wire attached to the stent was made of gold. Since this part constituted a relatively small proportion of the composition of the entire stent, it was excluded from the stent modelling. In this gold portion of the stent, the effect of the dose difference on the entire volume was small; however, there was a difference of $5 \%$ for the point dose in the TPS. In future studies, a simulation using accurate stent modelling which includes the complete composition of the stent will lead to more accurate dose analysis. The second limitation of the study was that dose perturbations were evaluated experimentally based on a single fraction with AP/PA fields. Actual clinical treatment consists of varying beam numbers and incident beam angles. In contrast, this study would have produced extreme results under relatively simple conditions. However, actual treatments are complicated by using multiple beams from multiple angles. Therefore, it is expected that the results will be reduced rather than the difference in the results performed according to the beam incidence direction and number. In order to obtain more accurate results, we will include patient data in our future studies of proton beams and photon beams which include the use of stents. 


\section{Conclusions}

This study confirmed that there was a difference in the dose delivered within the target volume by using $\mathrm{MC}$ simulation, which could not confirm the dose perturbation in the target volume even in the presence of the metal stent in the TPS calculation. Through MC simulations of photon and proton plans, we demonstrated that there are differences in the dosimetric parameters of the target volume as compared with those without a stent, for the positions and angles of the stent inserted into the phantom. Furthermore, we found that the stent caused a dose reduction rather than a dose increase of the target volume. In particular, this phenomenon appeared more clearly in the proton beam than in the photon beam.

Based on the results obtained from the MC simulation, we believe that delivery of the treatment beam avoiding the stent is the best method to prevent underdose and overdose in the target volume. However, we recommend that it is important to reduce the effect by using as many fractions and beams as possible, in order to reduce the effect of the stent when the beam is delivered.

\section{Abbreviations}

TPS: treatment planning system; MC: Monte Carlo; AP: anterior-posterior; PA: posterior-anterior; DVH: dose volume histogram; HCC: hepatocellular carcinoma; RT: radiotherapy; VMAT: volumetric modulated arc therapy; PBT: proton beam therapy; High-Z: high atomic number; SEMS: Self-expanding metal stents; ROI: region of interest; CT: computed tomography; TLD: thermoluminescent dosimeter; OSLD: optically stimulated luminescent dosimeter; IAEA: International Atomic Energy Agency; $D_{\text {max }}$ : maximum dose; $\mathrm{D}_{\text {min }}$ : minimum dose; $\mathrm{D}_{\text {mean }}$ : mean dose; DICOM: digital imaging and communications in medicine; HU: Hounsfield unit;

\section{Declarations}

\section{Ethics approval and consent to participate}

All procedures performed in studies involving a human participants was in accordance with the ethical standards of the institutional research committee. For this type of study, formal consent is not required.

\section{Consent for publication}

Not applicable

\section{Availability of data and material}

The datasets supporting the study conclusions are included within this manuscript.

\section{Competing interests}


B Lee, S Cho, HC Park, SW Kang, JS Kim, JB Chung declare that they have no competing interests.

\section{Funding}

This work was supported by the National Research Foundation of Korea (NRF) grant funded by the Korea Government (Ministry of Science and ICT, MSIT) (No. 2018R1D1A1B07049159 and 2020R1C1C100936611)

\section{Author's contributions}

JB Chung supervised the project, had given valuable advices on the proceeding of this work, and designed the concept and the experiment method of the research. B Lee, S Cho, HC Park and JS Kim supported on data collection and the characterization of the sample. B Lee and SW Kang performed the experiment and data analysis. B Lee and SW Kang wrote the paper and prepared Figures and Tables. JB Chung revised the manuscript. All authors have reviewed the final manuscript.

\section{Acknowledgements}

Not applicable

\section{References}

1. Kim DW, Chung K, Chung WK, Bae SH, Shin DO, Hong S, et al. Risk of secondary cancers from scattered radiation during intensity-modulated radiotherapies for hepatocellular carcinoma. Radiat Oncol. 2014:9:109.

2. Okuda K, Ohtsuki T, Obata H, Tomimatsu M, Okazaki N, Hasegawa $\mathrm{H}$, et al. Natural history of hepatocellular carcinoma and prognosis in relation to treatment. Study of 850 patients. Cancer. 1985:56(4):918-28.

3. Song TJ, Ip EW, Fong Y. Hepatocellular carcinoma: current surgical management. Gastroenterology. 2004:127:S248-60.

4. Emami B, Lyman J, Brown A, Coia L, Goitein M, Munzenrider JE, et al. Tolerance of normal tissue to therapeutic irradiation. Int J Radiat Oncol Biol Phys. 1991:21(1):109-22.

5. Yoo GS, Yu JI, Park HC. Proton therapy for hepatocellular carcinoma: Current knowledges and future perspectives. World J Gastroenterol. 2018:24(28):3090-100.

6. Yoo GS, Yu Jl, Cho S, Jung SH, Han Y, Park S, et al. Comparison of clinical outcomes between passive scattering versus pencil-beam scanning proton beam therapy for hepatocellular carcinoma. Radiother Oncol. 2020:146:187-93.

7. Jalaj S, Lee SY, McGaw C, John BK, Li Z, Awad ZT, et al. Proton radiotherapy dose perturbations caused by esophageal stents of varying material composition are negligible in an experimental model. Endosc Int Open. 2015:3(1):E46-50. 
8. Herrmann R, Carl J, Jakel O, Bassler N, Petersen JB. Investigation of the dosimetric impact of a Ni-Ti fiducial marker in carbon ion and proton beams. Acta Oncol. 2010:49(7):1160-4.

9. Jakel 0 , Reiss P. The influence of metal artefacts on the range of ion beams. Phys Med Biol. 2007;52(3):635-44.

10. Carl J, Nielsen J, Holmberg M, Hojkjaer Larsen E, Fabrin K, Fisker RV. A new fiducial marker for Imageguided radiotherapy of prostate cancer: clinical experience. Acta Oncol. 2008:47(7):1358-66.

11. Li XA, Chibani O, Greenwald B, Suntharalingam M. Radiotherapy dose perturbation of metallic esophageal stents. Int J Radiat Oncol Biol Phys. 2002:54(4):1276-85.

12. Chen YK, Schefter TE, Newman F. Esophageal cancer patients undergoing external beam radiation after placement of self-expandable metal stents: is there a risk of radiation dose enhancement? Gastrointest Endosc. 2011:73(6):1109-14.

13. Atwood TF, Hsu A, Ogara MM, Luba DG, Tamler BJ, Disario JA, et al. Radiotherapy dose perturbation of esophageal stents examined in an experimental model. Int J Radiat Oncol Biol Phys. 2012:82(5):1659-64.

14. Evans AJ, Lee DY, Jain AK, Razi SS, Park K, Schwartz GS, et al. The effect of metallic tracheal stents on radiation dose in the airway and surrounding tissues. J Surg Res. 2014:189(1):1-6.

15. Newhauser W, Fontenot J, Koch N, Dong L, Lee A, Zheng Y, et al. Monte Carlo simulations of the dosimetric impact of radiopaque fiducial markers for proton radiotherapy of the prostate. Phys Med Biol. 2007:520):2937-52.

16. Kim DH, Cho S, Jo K, Shin E, Hong CS, Han Y, et al. Proton range verification in inhomogeneous tissue: Treatment planning system vs. measurement vs. Monte Carlo simulation. PLoS One. 2018:13(3):e0193904.

17. Lee B, Jeong S, Chung K, Yoon M, Park HC, Han Y, et al. Feasibility of a GATE Monte Carlo platform in a clinical pretreatment QA system for VMAT treatment plans using TrueBeam with an HD120 multileaf collimator. J Appl Clin Med Phys. 2019:20(10):101-10.

18. Lee C, Lee S, Lee SJ, Song H, Kim DH, Cho S, et al. Monte Carlo simulation of secondary neutron dose for scanning proton therapy using FLUKA. PLoS One. 2017:12(10):e0186544.

19. Medical Information Processing Laboratory. Cost-effective and Com-pact Cluster Design for MonteCarlo Simulation. http://mipl.korea.ac.kr/cocomon/

20. Welsh J, Gomez D, Palmer MB, Riley BA, Mayankkumar AV, Komaki R, et al. Intensity-modulated proton therapy further reduces normal tissue exposure during definitive therapy for locally advanced distal esophageal tumors: a dosimetric study. Int J Radiat Oncol Biol Phys. 2011:81(5):1336-42.

21. Bom V, Joulaeizadeh L, Beekman F. Real-time prompt y monitoring in spot-scanning proton therapy using imaging through a knife-edge-shaped slit. Phys Med Biol. 2012:57(2):297-308.

22. Chow JC, Grigorov GN. Dose measurements near a non-radioactive gold seed using radiographic film. Phys Med Biol. 2005;50(18):N227-34. 
23. Chow JC, Grigorov GN. Monte Carlo simulations of dose near a nonradioactive gold seed. Med Phys. 2006;33(12):4614-21.

\section{Figures}

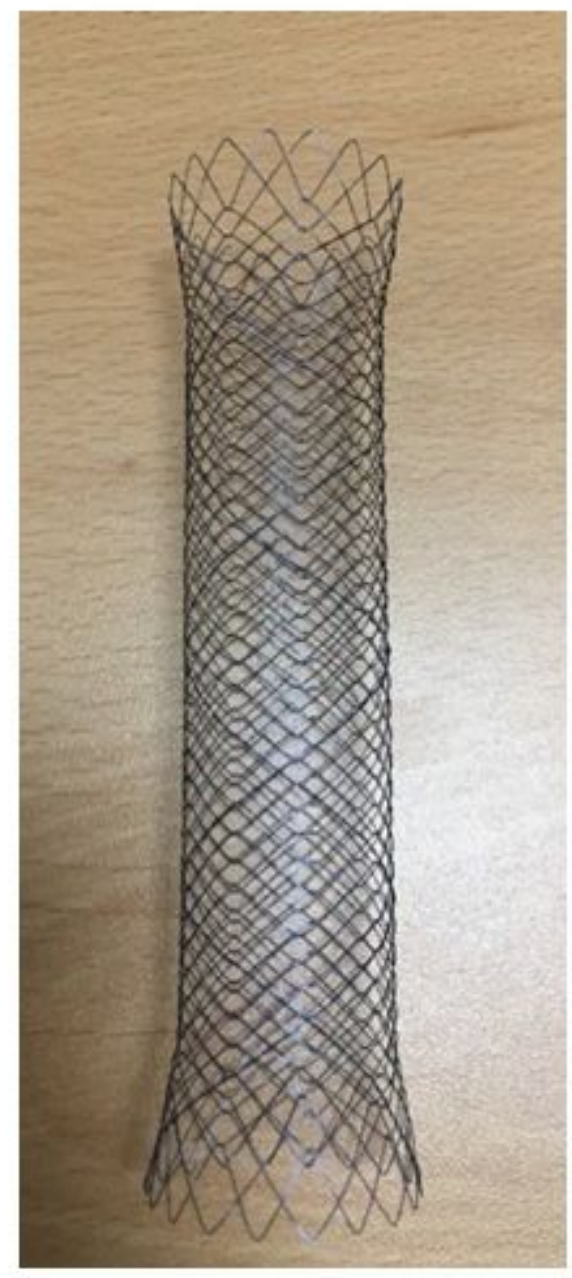

(a)

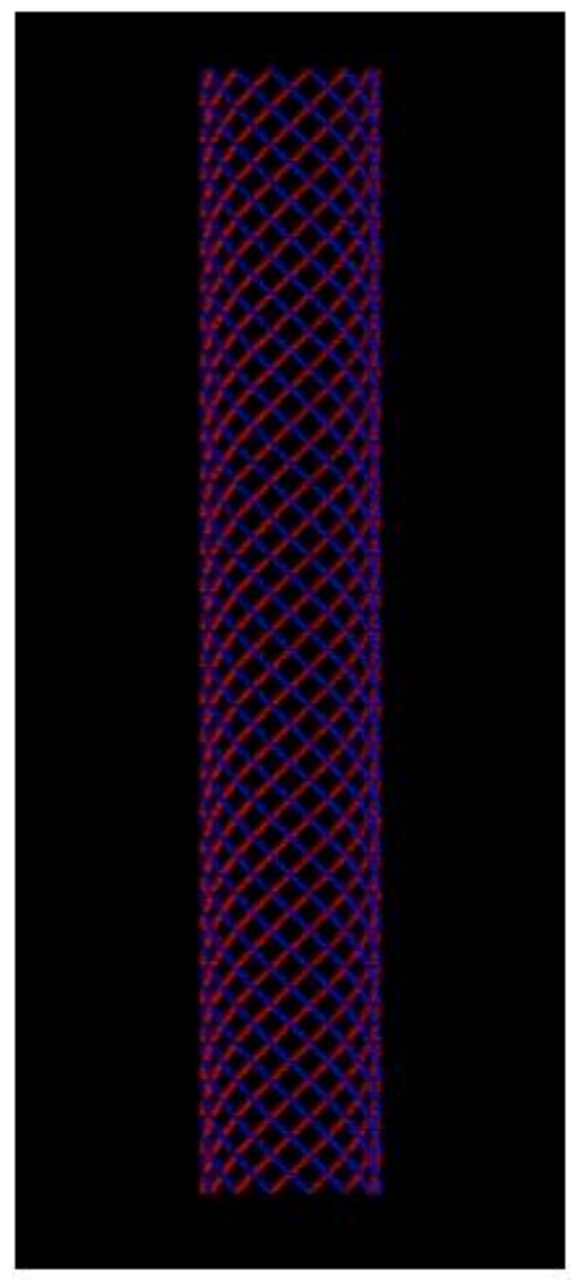

(b)

\section{Figure 1}

Metal stent (a) used in clinical practice and metal stent (b) modeled by MC simulation for dose calculation.

\section{Figure 2}

Planning schematic diagram to evaluate the effect of dosimetry parameters by metal stents by constructing the AP/PA fields. 


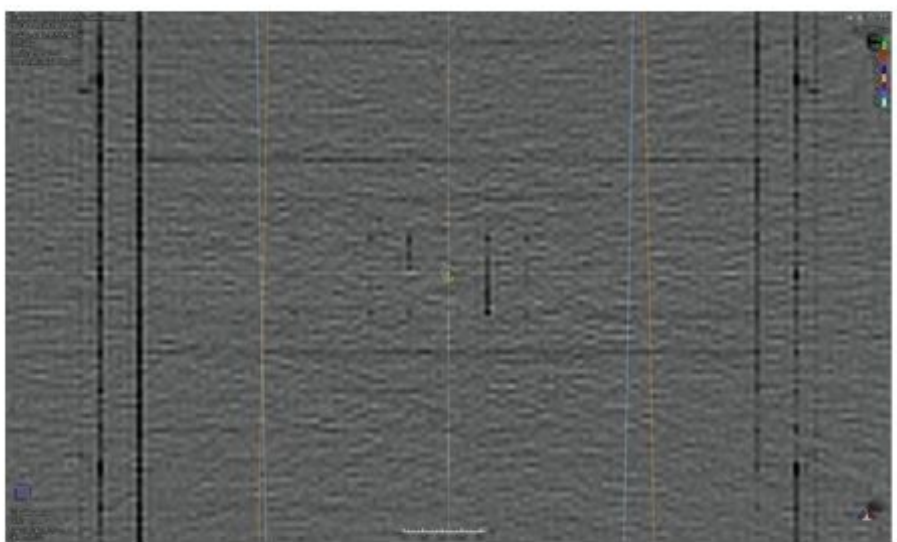

(a)

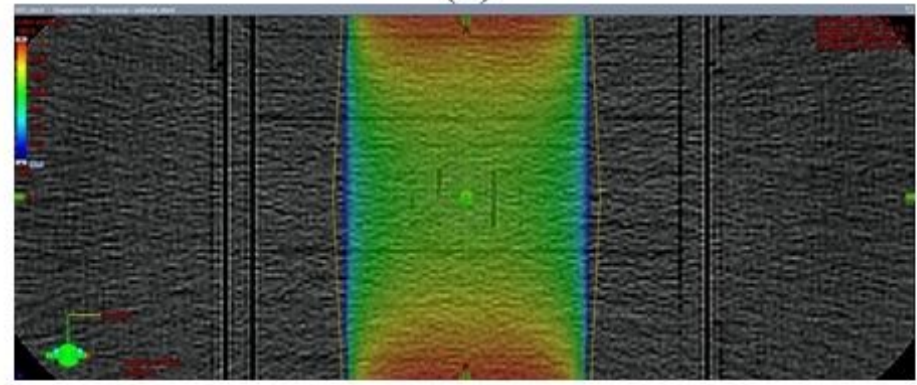

(c)

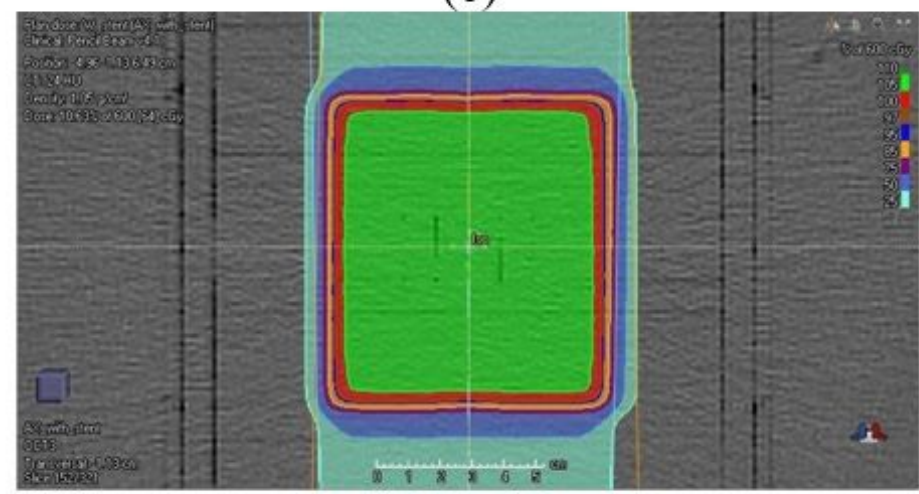

(e)

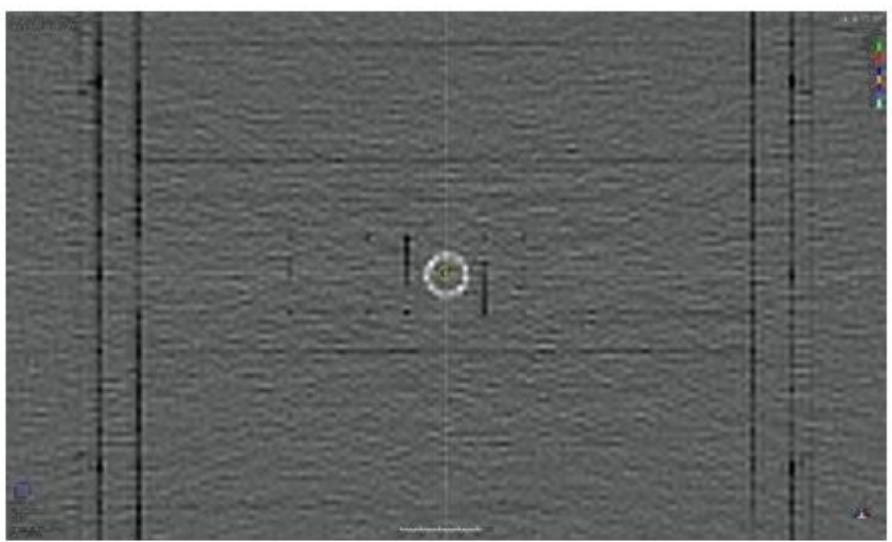

(b)

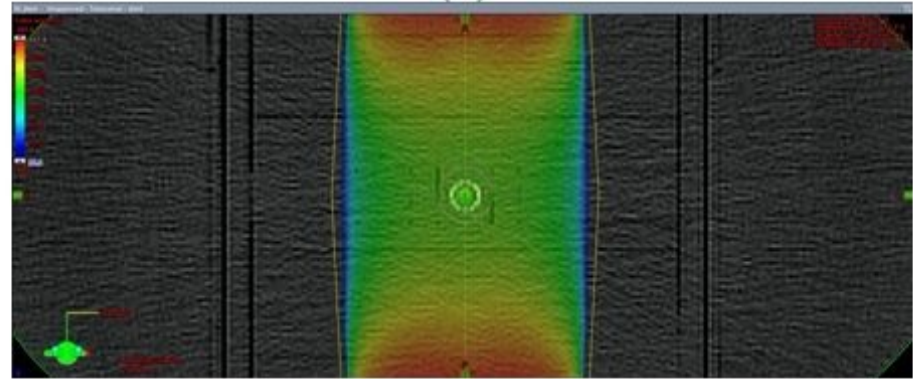

(d)

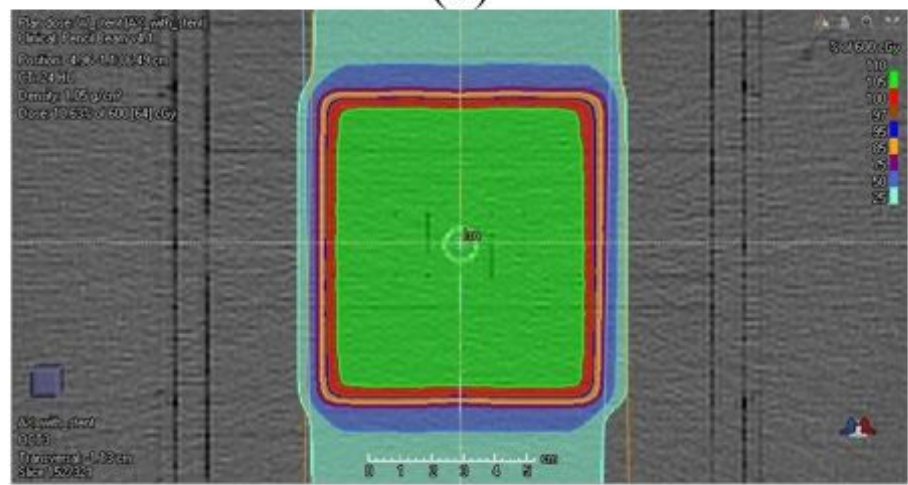

(f)

\section{Figure 3}

Scanned phantom image (a) without the metal stent and dose distributions calculated from TPSs for photon (c) and proton (e) plans, and scanned phantom image (b) with the metal stent and dose distributions calculated from TPSs for photon (d) and proton (e) plans. 


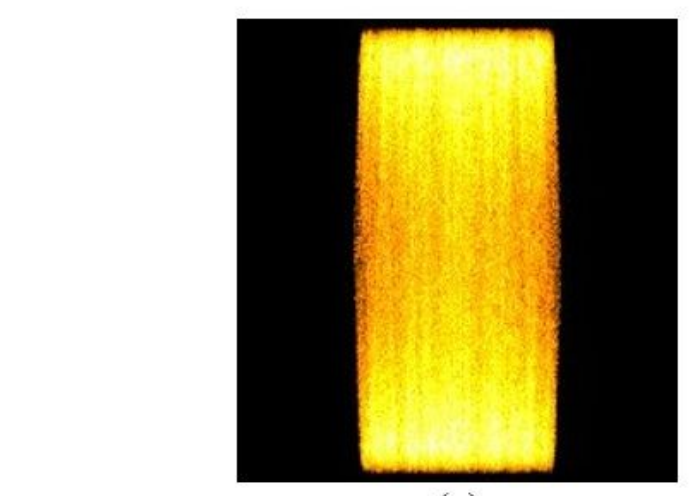

(a)

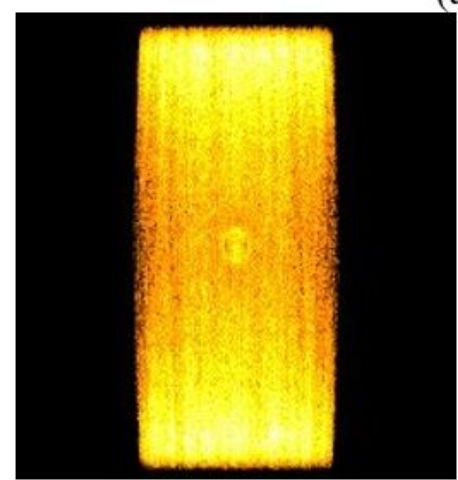

(d)

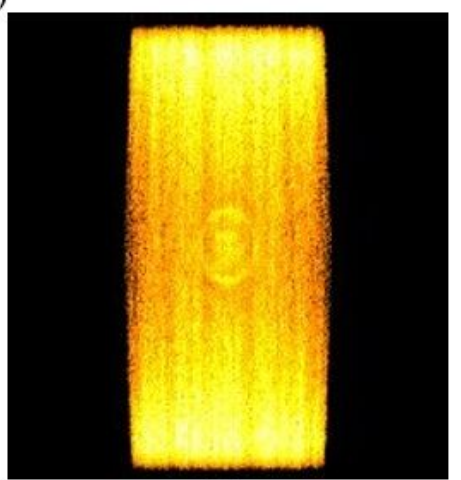

(e)

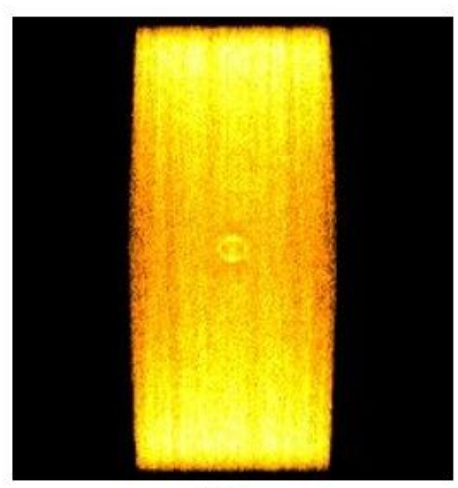

(b)

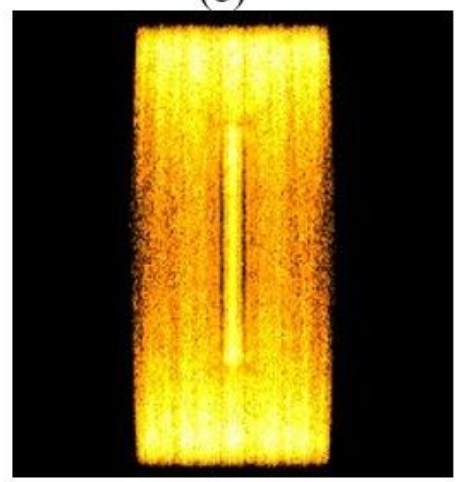

(f)

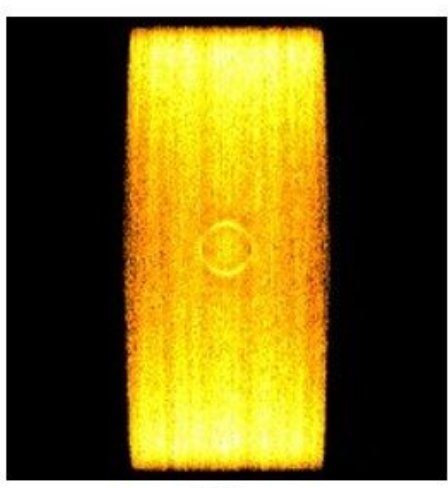

(c)

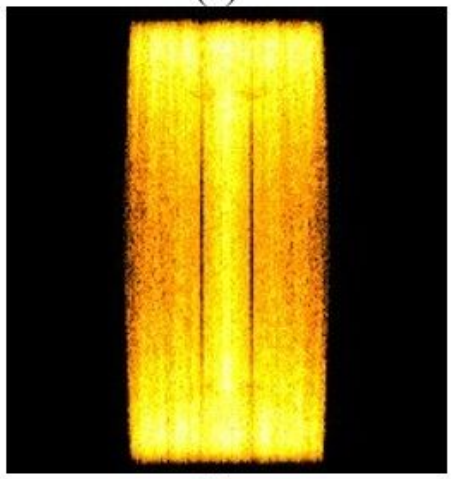

(g)

Figure 4

Axial dose distributions calculated by MC simulation for the photon beam. Dose distribution without the metal stent (a), dose distributions calculated from the GB stent (b) and GI stent (c) placed at an angle of $0^{\circ}$, dose distributions calculated from the GB stent (d) and GI stent (e) placed at angle of $45^{\circ}$, and dose distributions calculated for the GB stent (f) and GI stent (g) placed at an angle of $90^{\circ}$. 




(a)

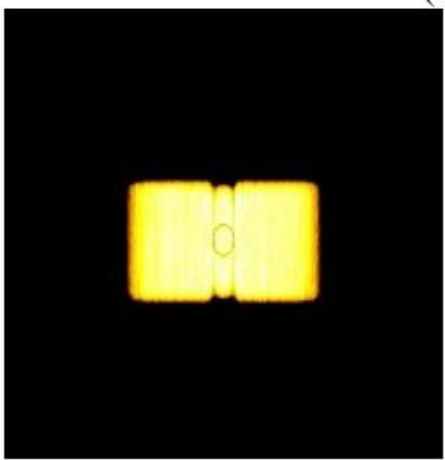

(d)

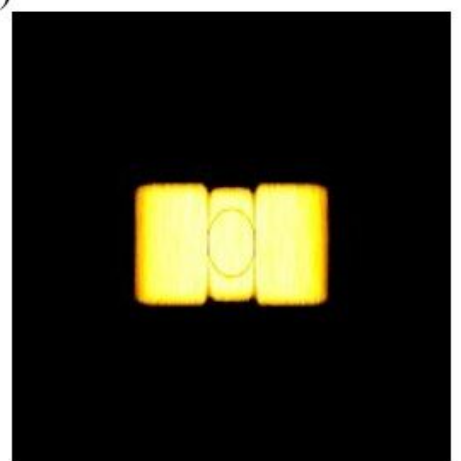

(e)

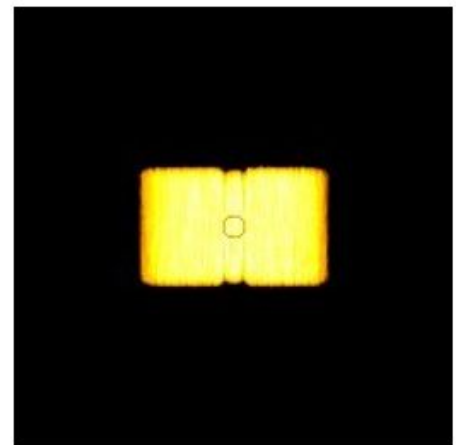

(b)

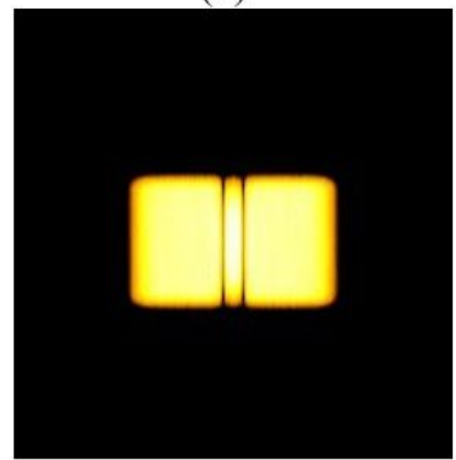

(f)

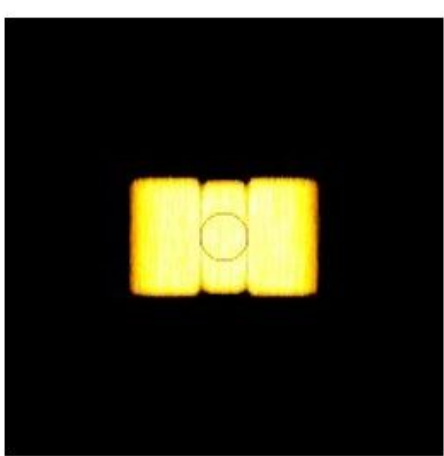

(c)

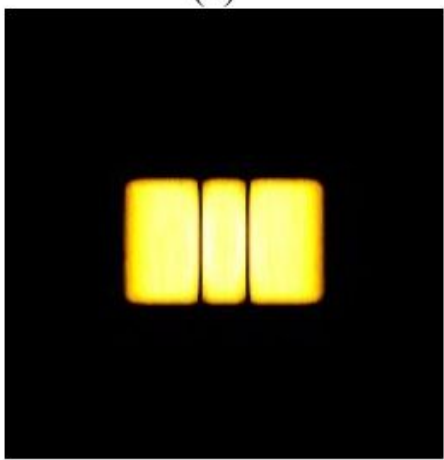

(g)

\section{Figure 5}

Axial dose distributions calculated by $\mathrm{MC}$ simulation for the proton beam. Dose distribution without the metal stent (a), dose distributions calculated from the GB stent (b) and GI stent (c) placed at an angle of $0^{\circ}$, dose distributions calculated from the GB stent (d) and GI stent (e) placed at angle of $45^{\circ}$, and dose distributions calculated for the GB stent (f) and GI stent ( $g$ ) placed at an angle of $90^{\circ}$. 


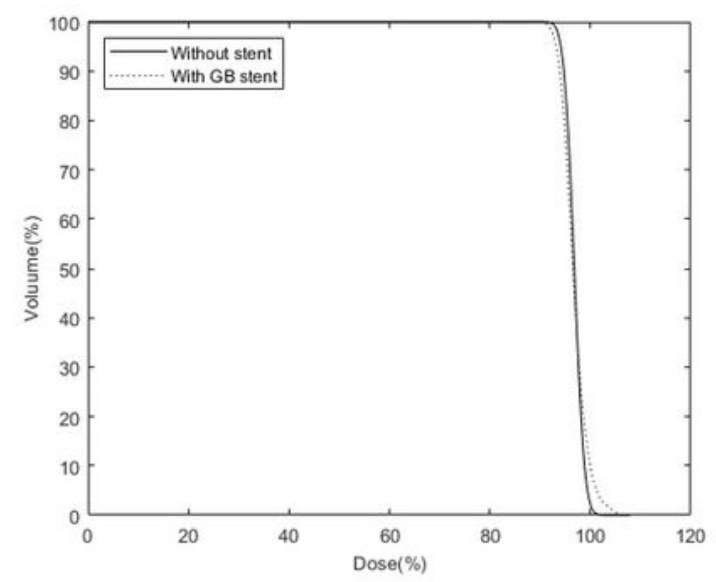

(a)

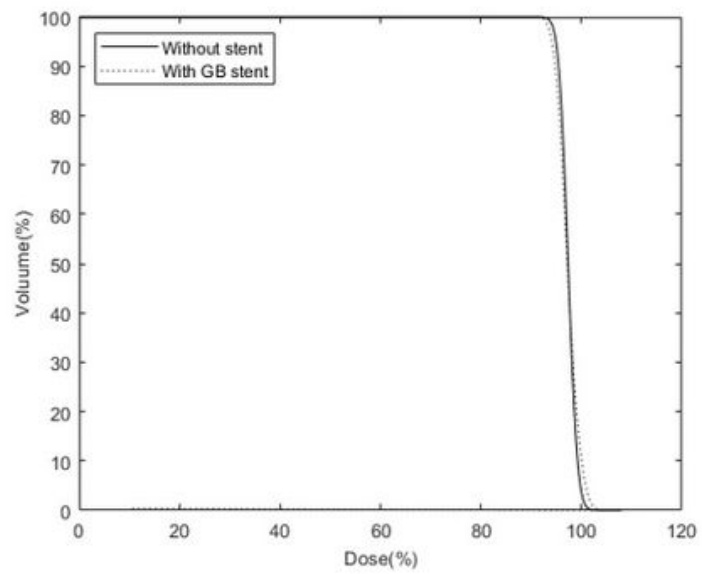

(c)

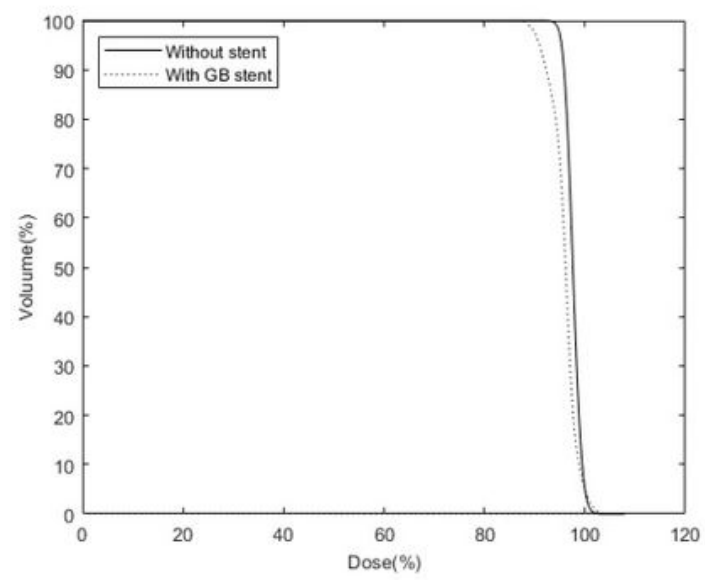

(e)

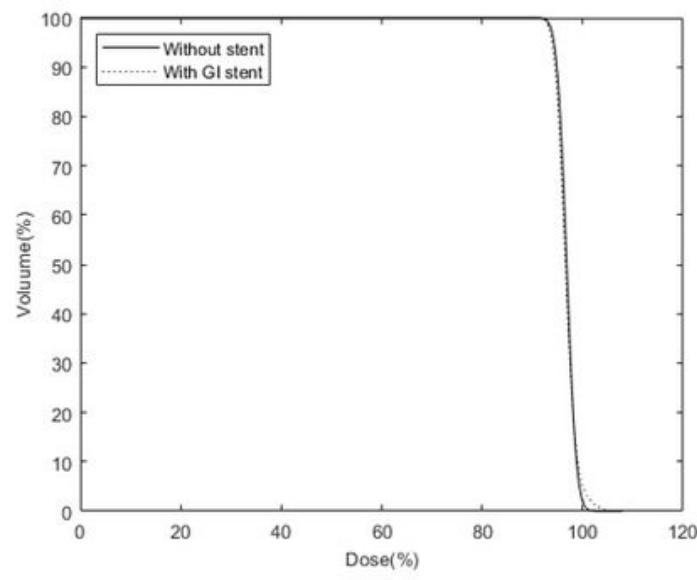

(b)

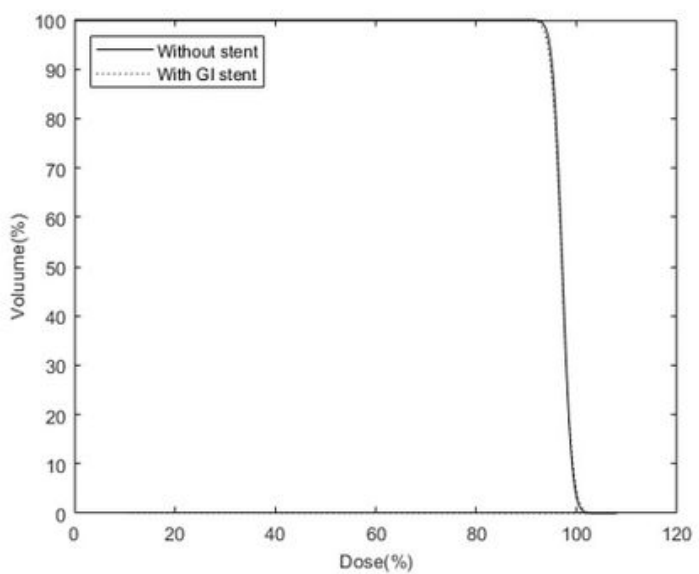

(d)

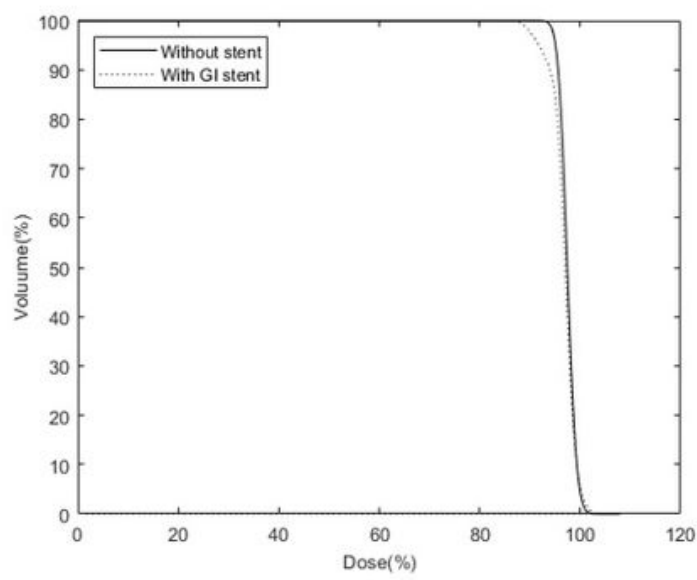

(f)

\section{Figure 6}

DVH according to the stent's position and angle compared to no-stent for the photon beam. DVHs calculated from the GB stent (a) and GI stent (b) placed at an angle of $0^{\circ}$, DVHs calculated from the GB stent (c) and GI stent (d) placed at an angle of $45^{\circ}$, and DVHs calculated from the GB stent (e) and GI stent (f) placed at an angle of $90^{\circ}$. 


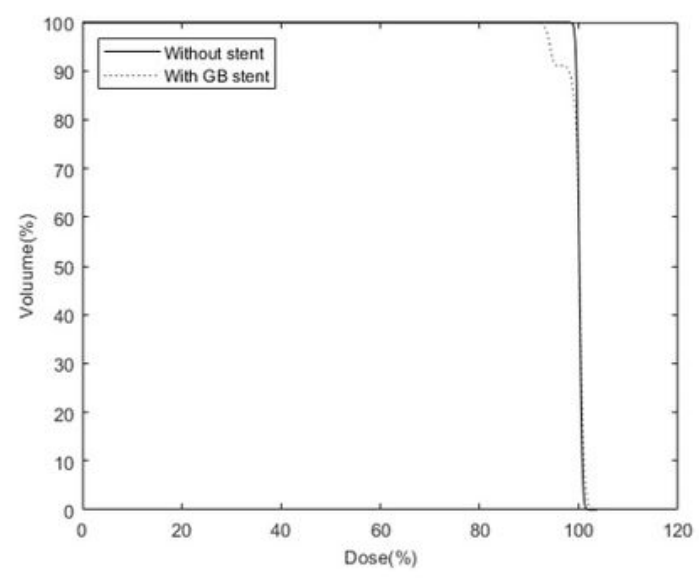

(a)

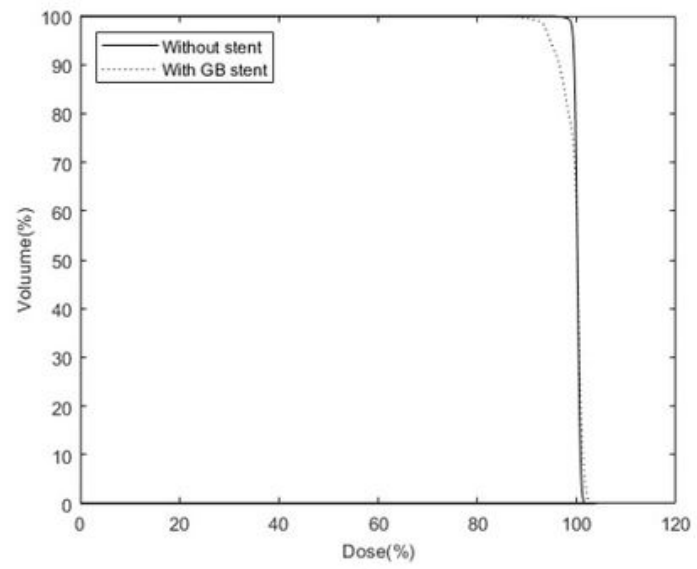

(c)

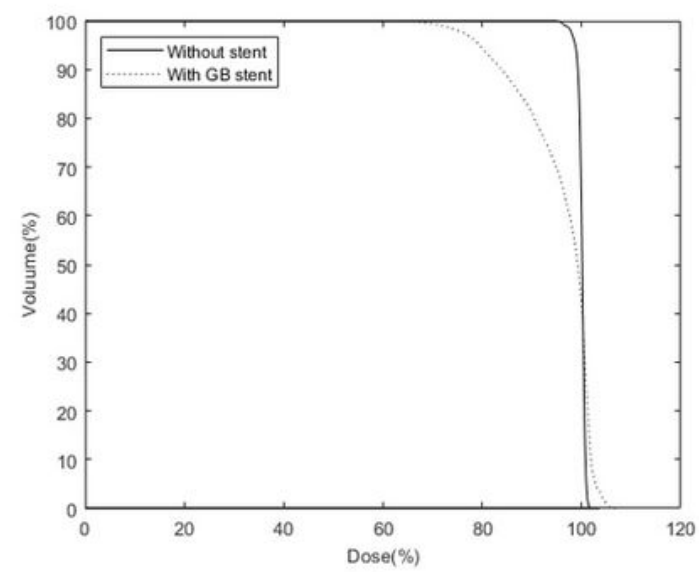

(e)

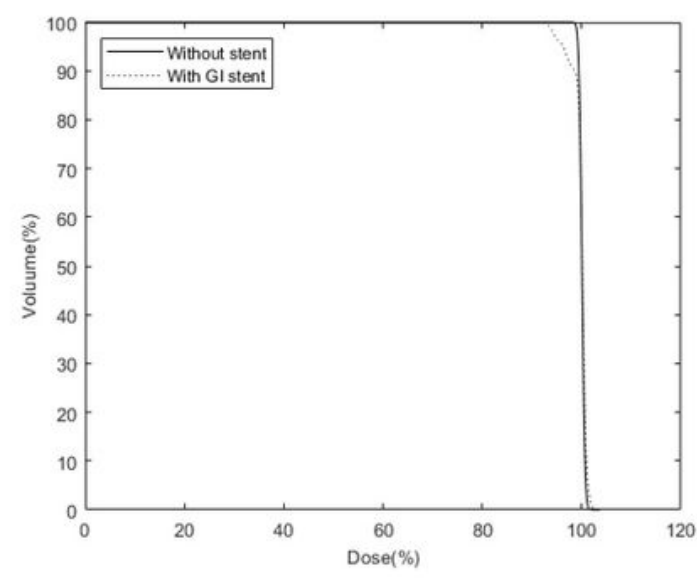

(b)

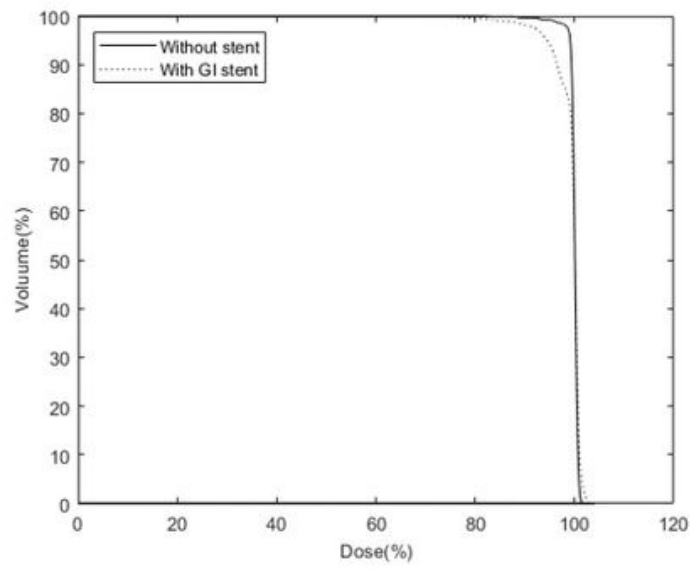

(d)

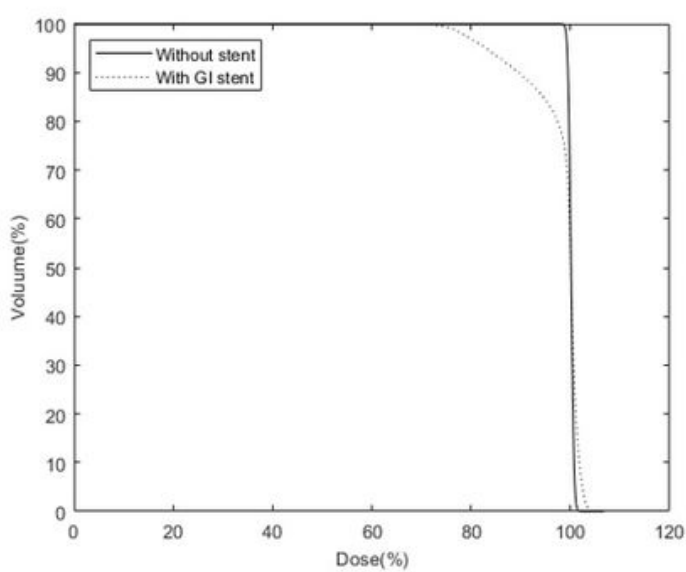

(f)

\section{Figure 7}

DVH according to the stent's position and angle compared to no-stent for the photon beam. DVHs calculated from the GB stent (a) and GI stent (b) placed at an angle of $0^{\circ}$, DVHs calculated from the GB stent (c) and GI stent (d) placed at an angle of $45^{\circ}$, and DVHs calculated from the GB stent (e) and GI stent (f) placed at an angle of $90^{\circ}$. 